\title{
Difficulty in evoking odor images: The role of odor naming
}

\author{
Richard J. Stevenson, Trevor I. Case, and Mehmet Mahmut \\ Macquarie University, Sydney, Australia
}

\begin{abstract}
Many studies report that people have difficulty in evoking odor images. In this article, we explore whether this results from another commonly observed phenomenon, difficulty in naming odors. In Experiment 1, participants both named and attempted to imagine either odors or their common visual referents. More-difficult-toname odors were reported as being more difficult to evoke as olfactory images, in comparison with the visual condition. In Experiment 2, participants received training prior to forming odor images and naming the same set of odors. As in Experiment 1, more-difficult-to-name odors were harder to imagine, but participants who had learned the odor names during training were significantly better, by their own report, at imagining many of these stimuli, relative to participants who were either exposed to the odors, exposed to their names, or who received no pretraining. In sum, these experiments suggest that odor naming may account for some of the difficulty reported by participants when attempting to evoke odor images; we discuss an associative basis for this effect.
\end{abstract}

Most participants report finding it easy to form a visual image. However, when participants are asked to form an olfactory image - that is, to imagine a smell - many describe the experience of evoking the smell to be more difficult and less vivid than a visual image. Why should this be so? In this article, we explore a possibility that has not been widely considered. To name an odor is harder than to name its visual referent. In many odor imagery studies, participants are told to evoke an olfactory image by the provision of its verbal referent: "Imagine lemon." Our contention here, spelled out more fully below, is that the limitation that is observed going from an olfactory percept/memory to its name also applies the other waythat is, going from the name to an olfactory memory/ percept. Consequently, we might expect a relationship to emerge between the ability to name a set of odorants and the self-reported capacity of participants to form olfactory images to the verbal referents of that same odor set. Similarly, we might expect that training to name a set of odors would improve participants' self-reported capacity to evoke olfactory images of that set. We explored these possibilities in the two experiments reported here.

That participants report difficulty in forming odor images is a well-established finding. Using a questionnaire approach, Sheehan (1967) found that participants reported less vivid images from the olfactory modality in comparison with all other modalities, a finding replicated in several other studies (e.g., Ashton \& White, 1980; White, Ashton, \& Law, 1978) and across cultures (Marsella \& Quijano, 1974). Similarly, Lawless (1997) asked participants how often they had experienced imagery in a range of modalities. Experiences of olfactory imagery were the least frequent and the most likely never to have been experienced (see also Brower, 1947; Lindauer, 1969). The obvious question, as we noted above, is why should phenomenal odor imagery be so poor?

Several possibilities may be advanced to answer this question. The most obvious is that we are unable to form olfactory images, and that what is reported results from experimental demand. This possibility appears unlikely for four reasons. First, there is a growing body of experimental evidence that suggests that imagining an odor can subsequently affect a real olfactory task, such as detecting an odor at threshold, for example (Djordjevic, Zatorre, \& Jones-Gotman, 2004; Djordjevic, Zatorre, Petrides, \& Jones-Gotman, 2004). Although we cannot be sure that participants utilize phenomenal imagery to complete such tasks, studies such as these offer qualified support for the existence of an odor imagery capacity. Second, although there are many reports of failures to obtain imagery effects (see Stevenson \& Case, 2005b), it is a characteristic of these null results that participants were never taught to associate the odorant with its verbal (or visual) referent. Third, many participants spontaneously sniff when asked to form an odor image. These sniffs tend to be larger for pleasant than for unpleasant imagined odors (Bensafi et al., 2003), indicating a sensorimotor reenactment of behavior present during normal smelling. Fourth, there is phenomenological evidence, relating to olfactory hallucinations, that people with no psychiatric ailment (e.g., migraine, temporal lobe epilepsy) have reported and then acted upon a hallucinated smell (e.g., Crosley \& Dhamoon, 1983; Daly, 1958). In sum, these findings are consistent with humans having the capacity to form a mental image of an odor.

R. J. Stevenson, richard.stevenson@psy.mq.edu.au 
A second possibility relates to ecological aspects of olfactory perception. To most of us, even though we might enjoy the sensations of eating and drinking (via retronasal olfaction), loss of our sense of smell is not accompanied by the severe difficulties faced by the sight or hearing impaired. Thus, we might argue that the unimportance of olfaction might imply that its full potentiality (of which forming odor images might be part) is never realized in most participants. Evidence consistent with this possibility has been obtained. Olfactory professionals, such as flavorists and perfumers, report being better able to imagine odors than naive participants, although the absolute magnitude of this effect is not huge (Gilbert, Crouch, \& Kemp, 1998). In a similar vein, sight-impaired participants, who consequently tend to pay more attention to other sensory domains, report more dreams with olfactory content than sighted participants (Hurovitz, Dunn, Domhoff, \& Fiss, 1999). Finally, normal participants may sometimes lack the proper technique for forming olfactory images. Bensafi, Pouliot, and Sobel (2005) found that the greater the similarity between sniffing patterns obtained for real and imagined odors, the better the self-reported imagery success. Together, these findings suggest that a lack of practice at forming odor images, and olfaction's ecological insignificance in man, may work against success at odor imagery.

A third possibility, and the one considered in this report, is that impoverished odor imagery results in part from a difficulty in accessing odor memory. Accounts of visual imagery suggest that evocation may be a consequence of retrieval of information from a long-term visual store (e.g., Kosslyn, Ganis, \& Thompson, 2003). In many experiments exploring phenomenal imagery, the cue to forming the image is the object's verbal referent"imagine a lemon," for example. Whereas this might be quite an effective means of recovering a visual memory, it may not be an effective means of recovering an olfactory one. This argument is premised on the relationship between the sense experience produced by perceiving an object and the generation of its veridical label or name, although this would apply equally to any other associate of the odor, such as an autobiographical memory, for example. In vision, this is typically easy for normal participants, with everyday objects, but it is not easy for normal participants to provide the name for everyday odorants when other cues are absent (e.g., Cain, 1979; Desor \& Beauchamp, 1974; Lawless \& Engen, 1977). Odor naming, then, is especially hard.

Three explanations have been suggested to account for this frequently made observation. The first is that odors may often be misperceived, resulting in retrieval of the correct name for the misperception, but the wrong name as far as the experimenter is concerned (e.g., Cain \& Potts, 1996; Jonsson, Tchekhova, Lonner, \& Olsson, 2005). Second, it may not always be possible to activate the verbal label for the odor, even though its general class (e.g., fruity), its familiarity, and its hedonic aspects may be appreciated. Support for this possibility comes from the observation that naming odors can be rapidly improved by paired-associate learning (odor-name), some- thing that would be hard to do if naming failures resulted primarily from misperceptions (e.g., Cain, 1979; Desor \& Beauchamp, 1974). Finally, Lorig (1999) has suggested that attempting to name an odor actively interferes with information-processing pathways used by language. However, this possibility appears to be problematic, in that it is not consistent with the finding that teaching participants to name an odor improves performance. The position we take here is best summed up by Cain, de Wijk, Lulejian, Schiet, \& See (1998): "Unstable access to semantic information presumably largely governs performance at identification" (p. 320).

Our basic contention, then, is that the difficulty that participants encounter when trying to evoke an odor image, after being provided with its verbal referent, is the reciprocal of the problem they encounter when trying to name it. That is, just as they have unstable access to semantic information when experiencing an odor percept, they have unstable access to olfactory information when provided with the odor's verbal referent. Although this possibility has not been tested directly, there is some circumstantial evidence in its favor. As noted above, many of the successful experimental demonstrations of odor imagery, measured by their impact on actual odor perception, involved a pretraining phase in which participants were instructed to learn the relationship between the odor and its verbal or pictorial label (e.g., Algom \& Cain, 1991; Algom, Marks, \& Cain, 1993; Djordjevic, Zatorre, Petrides, \& JonesGotman, 2004; Stevenson \& Prescott, 1997). From a different perspective, Stevenson and Case (2005a), in a study of olfactory dreams, obtained a weak correlation between odor-naming ability and the VOIQ (Vividness of Olfactory Imagery Questionnaire).

The present study set out to explore directly the relationship between odor naming and phenomenal odor imagery. In Experiment 1, we examined participants' ability to form images for a set of odors that they also named. In addition, another set of participants was asked to name and imagine pictorial representations of the odors' common visual referents, so that we could assess the degree to which any relationship between odor imagery and naming was not just a consequence of some peculiarity of the stimulus (e.g., persimmons may be harder to imagine in both modalities than tomatoes). In Experiment 2, we examined whether (1) learning to name a set of odors, (2) mere exposure to them, (3) mere exposure to their names, or (4) no pretraining, would differentially affect imagery ability for these stimuli. We predicted that learning odor names would be most effective. Our dependent variables here relied primarily on self-report. In particular, we were interested in difficulty of evocation and vividness. These variables were chosen because they have consistently been identified as being poorer for olfaction in comparison with the other senses (see above). The advantage of this method is that it allows us to ask directly about the most salient aspect of imagery - its phenomenal quality. Needless to say, such reports may suffer from various biases (see Richardson, 1999); however, several authors have reported positive correlations between such self-reports and measures of olfactory imagery not reliant on this form of measure 
(e.g., Bensafi et al., 2005; Djordjevic, Zatorre, Petrides, \& Jones-Gotman, 2004; Lyman \& McDaniels, 1990). More generally, in the visual imagery literature, where this relationship has been studied extensively, a modest but appreciable correlation $(r=.27)$ has been obtained between these variables, across studies (McKelvie, 1995).

\section{EXPERIMENT 1}

In Experiment 1, participants were assigned to either an olfactory or a visual condition, with half of the participants in each condition being asked to form mental images (olfactory or visual, respectively) first and to name the same stimuli second, or vice versa. Odors were selected to cover a range from easy-to-name to difficult-toname, on the basis of previous published and unpublished data from our laboratory (e.g., Stevenson \& Case, 2005a). The pictorial stimuli were chosen to represent common sources of the odors used in the olfactory condition, and were reproduced in color on A4-sized paper. In addition, participants' general propensity to form visual and olfactory images was measured using the VVIQ (Vividness of Visual Imagery Questionnaire; Marks, 1973) and the VOIQ; Gilbert et al., 1998), and their demand proneness was assessed with the Crowne and Marlow (1960) social desirability scale. All three measures were included to rule out the possibility that any differences between conditions might result from differences in these variables.

\section{Method}

\section{Participants}

Seventeen participants completed the olfactory condition (mean age $=21.5$ years, range $18-42$ years; 2 males, 15 females) and 19 participants completed the visual condition (mean age $=22.6$ years, range $=18-45$ years; 3 males, 16 females). No participant reported any olfactory deficit, and all of the participants had normal or corrected-to-normal vision. The participants completed the study for course credit.

\section{Stimuli}

Olfactory. The 15 odors used in Experiment 1 are detailed in Table 1. All of the stimuli were presented in 250-ml colored plastic sauce dispensers and were replaced weekly. The stimuli were refrig- erated between uses and were allowed to warm to room temperature prior to testing.

Visual. The 15 pictures used in Experiment 1 are also detailed in Table 1. The pictures of specific product containers had the words removed, but the distinctive shapes and colors of the containers and their labels were retained. Pictures were color reproductions on A4sized paper, presented one at a time in ring-top binders.

\section{Procedure}

Overview. Each participant attended two experimental sessions, one week apart. The participants were assigned by order of arrival to either the visual or the olfactory condition. Order of arrival also dictated whether or not they would receive the imagery task first and the naming task second, or vice versa. On the naming task, participants were asked to identify the stimulus (a picture/odor, depending on the condition), rate its familiarity, and say how much they liked/ disliked it. On the imagery task, they were asked to form images of the same set of stimuli, either olfactory or visual, depending on the condition. They then rated their difficulty of evocation, and how emotive the image was. Participants then completed the VOIQ, the VVIQ, and the Crowne-Marlow scale.

Naming task - odor. Participants completing the naming task were presented with their first odor and were allowed to smell it for as long as they wanted to. They were then instructed to provide the odor's name, and to guess if they did not know. They then rated how familiar the odor was (9-point category scale: 1 unfamiliar to 9 very familiar) and how much they liked or disliked it (9-point category scale: 1 pleasant, 5 neutral, 9 unpleasant). This process was then repeated for the remaining 14 odors. Presentation order was randomized for each participant, with one important caveat. The presentation order for the first participant in the odor-naming-first condition was the same as the order of presentation for the first participant in the odor-naming-second condition and for the first participant in the visual-naming-first condition and for the first participant in the visual-naming-second condition. Thus, presentation order was yoked across conditions.

Naming task-pictures. This was identical in all respects to the odor-naming condition, except that pictures were viewed and named.

Imagery task - odors. Participants were asked to "try to imagine the smell of $x$," where $x$ was one of the 15 odor names used in the naming part of the experiment. To do this, they were instructed to close their eyes and sniff, while trying to form a mental image of the smell. Both of these actions were employed in an attempt to maximize their chances of forming an image (Bensafi et al., 2003; Segal, 1971). When participants had managed to form an image, they were asked to open their eyes and complete the rating sheet. If, after $15 \mathrm{sec}$, they had not opened their eyes, they were instructed

Table 1

Odorants Used in Experiments 1 (and 2) and Visual Stimuli

\begin{tabular}{|c|c|c|c|}
\hline Odorant & Quantity (g) & Manufacturer & Pictorial Referent \\
\hline Coffee (ground) & 7.5 & Harris Kenyan & Coffee pot \\
\hline Vicks VapoRub & 7.0 & Vicks & Vicks VapoRub container \\
\hline Peanut butter & 12.0 & Home brand & Peanuts \\
\hline Vegemite & 7.4 & Kraft & Vegemite on toast \\
\hline Baby powder & 5.0 & Johnson \& Johnson & Baby powder container \\
\hline Tomato sauce & 7.0 & Heinz & Red sauce dispenser \\
\hline Lemon (citral) & 0.08 & Dragoco & Lemons \\
\hline Burnt wood & 0.04 & Sigma & Piece of burnt wood \\
\hline Garlic (crushed) & 3.5 & Home brand & Garlic cloves \\
\hline Vanilla (essence) & 0.25 & Queen & Vanilla ice cream \\
\hline Shoe polish & 8.0 & Kiwi & Tin of shoe polish (open) \\
\hline Almond (essence) & 0.1 & Dau Hanh Nhan & Almonds \\
\hline Oregano (oil) & 0.06 & Dragoco & Pizza topping (pizzaiola) \\
\hline Treacle & 7.0 & Blue Label & Jar of treacle (pouring) \\
\hline Ginger (dried) & 3.0 & Nature's Treats & Gingerbread man \\
\hline
\end{tabular}


to do so and then complete the rating sheet. Participants rated how difficult it had been to form their mental image (9-point category scale: 1 easy to evoke to 9 very difficult to evoke) and whether their mental image had a pleasant or unpleasant emotional tone (9-point category scale: 1 pleasant, 5 neutral, 9 unpleasant). This process was repeated for the remaining 14 odor names. Presentation order of the odor names used the same random sequence as in the naming task for that participant, so that the judgmental context remained the same.

Imagery task - pictures. This was identical to the odor imagery task, except that the participants were asked to form visual images of the items named by the experimenter.

Common tasks. The participants completed the two imagery scales (VVIQ and VOIQ) and the Crowne-Marlow social desirability scale, in that order.

\section{Analysis}

Naming data were scored (by R.J.S.) to a strict, predetermined criterion, as used before by Stevenson and Repacholi (2003), who reported high interrater agreement with this method. Names that matched those given by the experimenter were scored 2, those that were a close match were scored 1 , and all other responses were scored 0 . Close matches had to be specific objects, not object classes such as "fruit" (e.g., "onion," for garlic). The same scoring procedure was also adopted in Experiment 2. Finally, alpha was set at .05 in this experiment and in Experiment 2. All reported results are significant at this level except where stated.

\section{Results}

To examine the effect that naming had on imagery, the odor-naming data were first ranked according to correctness, from easy-to-name to hard-to-name. The first three stimuli were allocated to Level 1 (easy-to-name), the next three to Level 2, the next three to Level 3, the next three to Level 4, and the three hardest-to-name to Level 5 (see Table 2). There was one tie in the allocations (treacle and garlic), and this was resolved by assigning the odor with the smaller standard deviation to the easier-to-name level. The five naming levels for the visual referents were arranged in the same rank order as the odors, regardless of the identification scores they had received, so that the odor's visual referent would serve as its control. We note in passing that similar results to those reported below can be obtained by other partitioning methods (e.g., grouping imagery scores, etc., by the odors that each individual participant could and

Table 2

Odor and Picture Naming by Odor-Naming Difficulty Level in Experiment 1

\begin{tabular}{|c|c|c|}
\hline \multirow[b]{2}{*}{ Odor-Naming Difficulty (Stimuli) } & \multicolumn{2}{|c|}{ Mean \% Correct } \\
\hline & Odors & Pictures \\
\hline $\begin{array}{l}\text { Level } 1 \text { [Easiest] } \\
\quad \text { (Vicks, baby powder, tomato sauce) }\end{array}$ & 68.1 & 91.8 \\
\hline $\begin{array}{l}\text { Level } 2 \\
\quad \text { (Lemon, peanut butter, Vegemite) }\end{array}$ & 53.8 & 99.1 \\
\hline $\begin{array}{l}\text { Level } 3 \\
\quad \text { (Vanilla, coffee, treacle) }\end{array}$ & 34.3 & 64.0 \\
\hline $\begin{array}{l}\text { Level } 4 \\
\text { (Garlic, almond, shoe polish) }\end{array}$ & 19.5 & 94.5 \\
\hline $\begin{array}{l}\text { Level } 5 \text { [Hardest] } \\
\quad \text { (Oregano, ginger, burnt wood) }\end{array}$ & 12.3 & 66.3 \\
\hline Overall mean & 37.6 & 83.1 \\
\hline
\end{tabular}

could not name), but the method we have chosen to use here appears to yield a more complete picture.

\section{Odor and Visual Naming, Familiarity, and Hedonics, by Naming Difficulty Levels}

Odor- and picture-naming data were analyzed using a three-way repeated measures ANOVA with modality (olfactory vs. visual) and test order (naming first vs. imagery first) as between-participants factors and naming difficulty (easy to hard) as the within-participants factor. The ANOVA revealed an interaction between naming difficulty and modality $\left[F(4,128)=9.92, M S_{\mathrm{e}}=1.33\right.$; Huynh-Feldt $\varepsilon=1.0]$. As can be seen in Table 2, whereas naming performance fell markedly for the olfactory modality across levels (as intended), there was no consistent pattern for the visual stimuli. This was reflected in the significant linear $\left[F(1,32)=14.28, M S_{\mathrm{e}}=1.82\right]$ and quartic $\left[F(1,32)=25.21, M S_{\mathrm{e}}=0.91\right]$ trends for this interaction. The main effects of naming difficulty $[F(4,128)=$ 29.16, $M S_{\mathrm{e}}=1.33$; Huynh-Feldt $\varepsilon=1.0$ ] and modality $\left[F(1,32)=117.20, M S_{\mathrm{e}}=2.80\right]$ were of lesser interest, given the interaction above.

The same ANOVA design was used on familiarity and hedonic ratings of the real odors and pictures. Because these were of lesser interest, they are reported in abbreviated form. For both familiarity and hedonic ratings, interactions were obtained between naming difficulty and modality, with main effects for naming difficulty and modality. Familiarity ratings were lower, overall, for the odors $(M=5.9)$ relative to the pictures $(M=7.4)$, and familiarity fell markedly with increasing naming difficulty, unlike with the visual stimuli. Hedonic ratings were more negative, overall, for the odors $(M=5.3)$ relative to the pictures $(M=4.0)$, and odor hedonic ratings became more negative with increasing difficulty in naming, unlike with the visual stimuli.

\section{Difficulty of Image Evocation}

Participants' imagery evocation difficulty ratings were organized by the five levels of odor-naming difficulty and analyzed with a three-way repeated measures ANOVA, with modality (olfactory vs. visual) and test order (naming first vs. imagery first) as between-participants factors and naming difficulty (easy to hard) as a within-participants factor. The ANOVA revealed a significant interaction between modality and naming difficulty $[F(4,128)=11.03$, $M S_{\mathrm{e}}=0.68$; Huynh-Feldt $\left.\varepsilon=1.0\right]$, with participants rating it progressively harder to evoke images as naming difficulty increased in the olfactory condition relative to the visual condition (see Figure 1). This interpretation was confirmed by the presence of a significant linear trend for this interaction $\left[F(1,32)=37.90, M S_{\mathrm{e}}=0.63\right]$. The interaction between modality and test order was also significant $\left[F(1,32)=5.31, M S_{\mathrm{e}}=6.09\right]$. Participants who received the odor-naming task first reported it as significantly easier to evoke their images $(M=4.0)$ than participants who made the odor imagery ratings first $[M=5.5$; $t(15)=2.41]$. Test order appeared to exert no effect on the visual condition $(t<1)$. There was also a three-way 
interaction between modality, naming difficulty, and test order $\left[F(4,128)=4.34, M S_{\mathrm{e}}=0.68\right.$; Huynh-Feldt $\varepsilon=$ $1.0]$. This appeared to result from the odor conditions, because a test of the contrast naming difficulty $\times$ test order in the visual condition was not significant. The same contrast in the olfactory condition, however, was $[F(4,60)=$ 2.61, $M S_{\mathrm{e}}=2.81$; Huynh-Feldt $\left.\varepsilon=1.0\right]$, and this had both quartic $\left[F(1,15)=9.18, M S_{\mathrm{e}}=0.62\right]$ and marginally significant linear $\left[F(1,15)=3.94, M S_{\mathrm{e}}=0.88, p=.066\right]$ trend components. Thus, whereas easier-to-name odors were somewhat harder to evoke for participants who had undertaken imagery first, a more complex relationship also underlay this pattern. There were also main effects for naming difficulty $\left[F(4,128)=7.80, M S_{\mathrm{e}}=0.68\right.$; Huynh-Feldt $\varepsilon=1.0]$ and modality $[F(1,32)=37.91$, $\left.M S_{\mathrm{e}}=6.09\right]$, but these were of lesser interest in the context of their interaction effects reported above. However, the modality effect indicates that participants, overall, reported greater difficulty in evoking odor images $(M=$ $4.7)$ than visual ones $(M=2.4)$.

\section{Hedonic Deviation of the Image}

The degree to which participants' imagery hedonic ratings differed absolutely from their hedonic ratings of the real stimuli was examined using the same three-way ANOVA. The ANOVA revealed a main effect of modality $\left[F(1,32)=9.43, M S_{\mathrm{e}}=1.45\right]$, in that odor images were more deviant from the hedonic rating applied to their real equivalent $(M=2.0)$ than visual images and their real equivalent $(M=1.5)$. There was an interaction between naming difficulty and modality $\left[F(4,128)=4.03, M S_{\mathrm{e}}=\right.$ 0.91 ; Huynh-Feldt $\varepsilon=1.0]$. However, there was no clear pattern of increasing hedonic deviation as odor-naming difficulty increased (i.e., no linear or quadratic trends).
Finally - of lesser interest because of the interactions reported above - there was a main effect of naming difficulty $\left[F(4,128)=2.81, M S_{\mathrm{e}}=0.91 ;\right.$ Huynh-Feldt $\left.\varepsilon=1.0\right]$.

\section{Predicting Odor-Image-Evocation Difficulty}

The unit for this analysis was the odor, so we used the data from all 17 participants in the olfactory condition. For each odor, we calculated its mean naming, familiarity, and hedonic rating scores. For its odor image, we calculated the mean ease-of-evocation score. In addition, we calculated the mean naming score for the odor's visual referent (using data from the visual condition). Easeof-odor-image-evocation score was then treated as the dependent variable, with real odor familiarity, naming, hedonics, and the naming score for the odor's visual referent as predictors. This regression model accounted for $54.4 \%$ of the variance (adjusted) in difficulty-of-imageevocation score $[F(4,10)=5.18]$. The best predictor was odor-naming score, which uniquely accounted for $39.3 \%$ of the variance (squared semipartial correlation coefficient), followed by odor hedonics $(17.3 \%)$, naming the visual referent $(6.1 \%)$, and familiarity $(<0.1 \%)$. The only significant independent predictors were odor naming $(t=$ $3.47)$ and hedonics $(t=2.30)$.

\section{Imagery and Social Desirability}

There were no significant differences in either selfreported visual or olfactory imagery, nor in social desirability, between conditions (ANOVA).

\section{Discussion}

Four findings emerged from this experiment. First, the more difficult it was to name an odor, the more difficult it was to evoke its image. Second, the visual referents of the

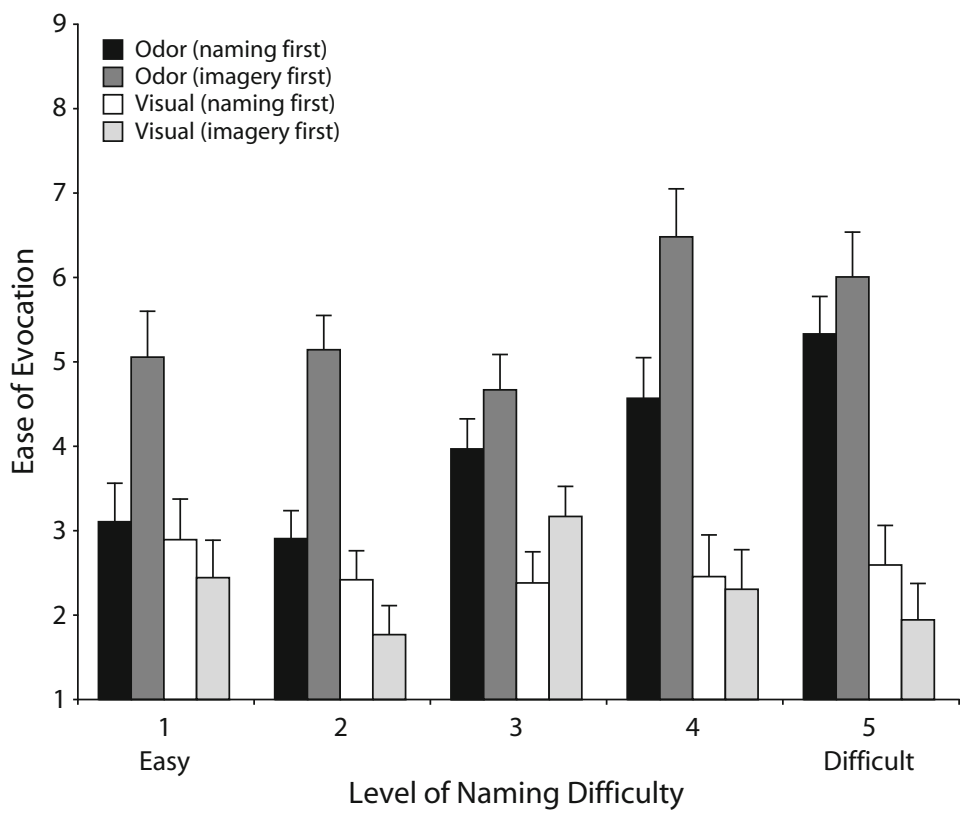

Figure 1: Mean ease of imagery evocation (and $S E$ ) for each of the four groups in Experiment 1, by odor-naming difficulty. 
olfactory stimuli did not behave in this way, suggesting that it was not the objects per se, but rather the difficulty that participants had in naming their olfactory source. Third, for odors, attempting to name them in the naming phase appeared to enhance the ease with which participants reported evoking their image. Fourth, under conditions in which the odor became the unit of analysis, difficulty in evoking an odor's image was best predicted by how hard its olfactory referent was to name, followed by its hedonic tone, but not by how difficult its visual referent was to name or by the odor's familiarity. The absence of odor familiarity as a predictor may reflect the often observed correlation between hedonic and familiarity ratings. In sum, these findings suggest that odor-naming difficulty is related to odor imagery difficulty. However, to establish whether this is causal requires demonstrating that learning odor names, but not other forms of exposure (or none at all), enhances phenomenal imagery. Experiment 2 set out to test this.

\section{EXPERIMENT 2}

As noted in the introduction, several successful demonstrations of the effect of odor imagery on an actual olfactory task have involved a prior training phase in which the association between the odor's visual or verbal referent and the percept has been strengthened. In the experiment reported here, we set out to test this explicitly, by determining whether training participants to associate a set of odors and names was more beneficial to their later reports of ease of evocation than (1) mere exposure to the odors, (2) exposure to the odor names but not the odors, or (3) no pretraining at all. In the introduction, we predicted that only odor naming would be beneficial. Finally, two further measures of imagery were used in this study: the length of time that participants shut their eyes while evoking the image, and ratings of imagery vividness. The former was included because, in Experiment 1, participants tended to open their eyes quite quickly when they had readily formed an image, so we felt it was worthwhile quantifying this. The latter was included because it has been used before in assessment of imagery ability.

\section{Method}

\section{Participants}

Forty-eight participants took part in Experiment 2, all of whom were naive to the experiment's purpose and had not taken part in Experiment 1. The four experimental groups had similar mean ages, which did not significantly differ (exposure to odor names [ETON] $M=22.5$ years, range 18-40 years, 3 males and 9 females; learning odor names $[\mathrm{LON}] M=24.3$ years, range $18-50$ years, 3 males and 9 females; mere exposure to odors [METO] $M=24.5$ years, range 19-43 years, 3 males and 9 females; no exposure [NEP] $M=$ 22.2 years, range $18-52$ years; 2 males and 10 females). Participants were paid AU $\$ 20$ for taking part.

\section{Stimuli}

The olfactory stimuli were the same as those used in Experiment 1 (see Table 1).

\section{Procedure}

Overview. The participants attended a training session and then, one week later, a testing session, except for those in the NEP condi- tion, who only attended the testing session. Training in the LON condition involved four blocks of odor-name learning, with each block composed of 15 trials, in which each participant attempted to name the target odorant and then received corrective feedback. Training in the METO condition involved the same training regime as in the LON condition, except that here, participants were asked to rate how much they liked or disliked each odor. Training in the ETON condition involved the same exposure regime as in the previous two conditions, but, crucially, only to the odor's name. In this case, no specific mention of odor was made, and the participants were simply asked how much they liked or disliked each word (e.g., "lemon"). The second session, the test phase, was identical for all of the participants. First, participants were asked to form odor images of each of the 15 stimuli and then, for each, to rate ease of evocation, vividness, and liking. The length of time they reportedly took to generate each image was also timed. Second, they completed the VOIQ and the Crowne-Marlow social desirability scale. Finally, they were presented with the 15 real odors (equivalent to those they had just tried to imagine) and were asked to name each one, judge its familiarity, and rate how much they liked or disliked it.

Training. Participants were assigned to each of the four groups on the basis of order of arrival. However, in the latter part of the experiment, we attempted to balance age and gender between the conditions, so some allocations were made on this basis.

In the LON condition, participants received four blocks of training trials, with each block composed of the 15 odors listed in Table 1. On each block, participants were asked the odor's name. They had to generate a response, and this was written down by the experimenter. If the response was correct, the experimenter said "That is correct." If the response was incorrect, the experimenter said "No. This odor is called $x$. Please say its name out loud." This process then continued for the remaining 14 odors in that block, with each odor presentation separated by a minimum of $15 \mathrm{sec}$. The second, third, and fourth blocks were identical in form, although a different random presentation order was used for each block. Different random presentation orders were used for each of the 12 participants who received this training regime.

In the METO condition, participants also received four blocks of trials, with each block composed of 15 odors. In this case, participants smelled each odor and then completed a 9-point hedonic rating scale (see Experiment 1). Each trial was separated by $15 \mathrm{sec}$. Presentation order was yoked to the LON condition, so that the first participant in the METO condition received the same presentation order.

In the ETON condition, participants again received four blocks of 15 trials. However, in this case, each trial was an odor name, which they had to read out loud and then rate, using a 9-point hedonic rating scale (see Experiment 1). Presentation order of the words was yoked to the LON and METO conditions, so that the first participant in the ETON condition received his or her odor names in the same random order as the first participant in the LON and METO conditions.

Testing. All of the participants (including those in the NEP condition, for whom testing was the first session attended) received an identical procedure. First, they were asked to imagine the smell of each of the 15 odors described in Table 1. The length of time they spent with their eyes closed, attempting to imagine the odor, was timed (up to a maximum of $15 \mathrm{sec}$ ), and when they opened their eyes, they were asked to complete three rating scales: how difficult they found the task, 1 (easy to imagine) to 9 (very difficult to imagine); how vivid, if at all, the odor image was, 1 (very vivid image) to 9 (no image present at all); and finally, whether it evoked any pleasant or unpleasant feelings, 1 (pleasant feeling), 5 (no particular feeling), 9 (unpleasant feeling). This process was then repeated for the remaining items. The participants' motivation to complete the task was rated by the experimenter after the participant had completed the remainder of the experiment. This was a purely subjective judgment by the experimenter, and was included to potentially identify participants who were disinterested and did not appear to be making a valid attempt at the various tasks. Order of presentation of the test items was random, but again was yoked across the four experimental conditions. 
All of the participants then completed the VOIQ and the CrowneMarlow social desirability scale. The final part of the test phase involved naming the 15 odors corresponding to those used in the imagery phase above (and to the odor stimuli used in the LON and METO training). For each odor, participants were asked to name the odor and to guess if necessary. They then rated the odor for familiarity and liking using the same scales as for Experiment 1. Again, at least $15 \mathrm{sec}$ separated each trial. Order of presentation was yoked to the imagery phase, in order to preserve the judgmental context.

\section{Results}

Most of the participants in the LON condition successfully acquired the names across the four blocks of trials (Block 1, $M=31.5 \%$ correct; Block 2, $M=70.9 \%$ correct; Block 3, $M=84.8 \%$ correct; Block 4, $M=86.7 \%$ correct; $n=11$ ). However, 1 participant was significantly below the mean (by $2 S D$ s) on every block and so was eliminated from further analysis on this basis.

The same basic approach used to analyze the data in Experiment 1 was adopted here. To examine the effect that naming had on imagery, the odor-naming data obtained in the final part of the test session were collapsed across all four experimental conditions (LON, ETON, METO, and NEP) and ranked in the same manner as described for Experiment 1 (see Table 3). There was one tie in the allocations (almond and burnt wood) and this was resolved by assigning the odor with the smaller standard deviation to the easier-to-name level.

\section{Odor Naming, Familiarity, and Hedonics, by Naming Difficulty Levels}

Naming data from the final part of the test session were analyzed using a three-way repeated measures ANOVA, with naming difficulty as a within-participants factor (easy to hard) and exposure (exposed to odors vs. not exposed to odors) and naming (odor names provided vs. odor names not provided) as between-participants factors. There were main effects of naming difficulty $\left[F(3.4,148.0)=42.78, M S_{\mathrm{e}}=2.25\right.$; Huynh-Feldt $\varepsilon=$ $0.86]$ and naming $\left[F(1,43)=33.44, M S_{\mathrm{e}}=4.77\right]$. These effects interacted $\left[F(3.4,148.0)=3.33, M S_{\mathrm{e}}=2.25\right.$; Huynh-Feldt $\varepsilon=0.86]$, with better performance for the moderately difficult-to-name odors in participants who had been provided with names relative to those who had not (see Table 3). Most important of all, though, was the interaction between naming and exposure $[F(1,43)=$ 7.86, $\left.M S_{\mathrm{e}}=4.77\right]$, which indicated, as expected, better performance from the participants who learned the odors' names, relative to the other conditions [LON vs. the other conditions, $t(45)=5.48]$.

The same ANOVA design was used on familiarity and hedonic ratings of the real odors. These were of lesser interest and are reported in brief. For familiarity, main effects of naming difficulty, naming, and an interaction between naming and exposure were obtained. Familiarity ratings were highest for easy-to-name odors and higher for groups that had received prior exposure. For hedonic ratings, the same effects were obtained. Odors were judged more favorably if their names had been learned.

\section{Evocation Difficulty, Vividness, and Time}

Participants, when asked to imagine each of these 15 odors, made both evocation difficulty ratings, as in Experiment 1 , and imagery vividness ratings, as well. In addition, the length of time that participants kept their eyes shut and sniffed while attempting to form the image was also timed (to a maximum of $15 \mathrm{sec}$ ). Thus, three measures were obtained of participants' reported difficulty and success in forming an image, and all of these measures significantly correlated with each other (median $r=$ .50 , range .22 to .89 ; correlation between each dependent variable at each level of naming difficulty [15 in total]). The time data were rescaled so that the maximum score (time in seconds) was 9, prior to analysis, so that there was a common dimension to all the dependent variables. These data were then entered into a four-way ANOVA, with measure (evocation difficulty, vividness, time) and naming difficulty (easy to hard) as within-participants factors and exposure (exposed to odors vs. not exposed to odors) and naming (odor names provided vs. odor names not provided) as between-participants factors.

As in Experiment 1, there was a main effect of naming difficulty $\left[F(4,172)=22.27, M S_{\mathrm{e}}=2.62\right.$; HuynhFeldt $\varepsilon=1.0]$, with easier-to-name odors reported as

Table 3

\begin{tabular}{|c|c|c|c|c|}
\hline \multirow[b]{2}{*}{ Odor-Naming Difficulty (Stimuli) } & & \multicolumn{2}{|c|}{ Mean \% Correct } & \\
\hline & $\mathrm{LON}$ & METO & ETON & NEP \\
\hline el 1 [Easiest] & & & & \\
\hline $\begin{array}{l}\text { (Vicks, baby powder, lemon) } \\
\text { evel } 2\end{array}$ & 93.9 & 70.8 & 88.8 & 75.0 \\
\hline $\begin{array}{l}\text { (Vanilla, tomato sauce, peanut butter) } \\
\text { evel } 3\end{array}$ & 87.9 & 58.3 & 66.7 & 54.2 \\
\hline $\begin{array}{l}\text { (Vegemite, coffee, shoe polish) } \\
\text { evel } 4\end{array}$ & 93.9 & 34.7 & 69.5 & 41.7 \\
\hline $\begin{array}{l}\text { (Garlic, almond, oregano) } \\
\text { evel } 5 \text { [Hardest] }\end{array}$ & 78.8 & 19.5 & 44.5 & 27.8 \\
\hline (Treacle, ginger, burnt wood) & 45.5 & 12.5 & 20.8 & 20.8 \\
\hline verall mean & 80.0 & 39.2 & 58.1 & 43.9 \\
\hline
\end{tabular}


easier to imagine, faster to evoke, and more vivid than hard-to-name odors, as indicated by the significant linear $\left[F(1,43)=51.91, M S_{\mathrm{e}}=3.73\right]$ and quadratic $[F(1,43)=$ 17.67, $\left.M S_{\mathrm{e}}=1.96\right]$ trends. Naming difficulty and measure $\left[F(5.6,243.0)=8.33, M S_{\mathrm{e}}=5.73\right.$; Huynh-Feldt $\varepsilon=0.71]$ interacted, with less striking changes over increasing naming difficulty for the time variable relative to the difficulty and vividness variables. There was also a main effect of measure $\left[F(1.2,50.8)=9.07, M S_{\mathrm{e}}=9.84\right.$; Huynh-Feldt $\varepsilon=0.59]$, which reflected the larger scores in the time-dependent variable, in comparison with the other variables.

The most important finding, however, was the threeway interaction between naming difficulty, naming, and exposure $\left[F(4,172)=2.67, M S_{\mathrm{e}}=2.62\right.$; Huynh-Feldt $\varepsilon=1.0]$, which is illustrated in Figures $2 \mathrm{~A}-2 \mathrm{D}$ (by group). Here it can be seen that self-reported imagery performance (ease of evocation, vividness, and time to form an image) was best in the LON condition, at least for the first three levels of difficulty (1-3), but not for the two most difficult levels (4 and 5). This interaction was examined in three ways. First, a further ANOVA was run to determine whether there were any differences in performance between the three groups that did not learn odor names (i.e., METO, ETON, and NEP). A three-way repeated measures ANOVA was conducted, with naming difficulty and measure as within-participants factors (as described above) and group (METO vs. ETON vs. NEP) as a between-participants factor. Crucially, there was no significant interaction between group and naming difficulty $(F=1.05)$, nor any other effect involving group. The other effects reported above (naming difficulty, measure, measure $\times$ naming difficulty) were still significant.

Because these three conditions, METO, ETON, and NEP, did not significantly differ from each other, they were combined and contrasted with the LON group in a second analysis (three-way ANOVA, with measure and naming difficulty as within-participants factors and group [LON vs. combined] as the between-participants factor). As above, naming difficulty, measure $(p<.06)$, and measure $\times$ naming difficulty were significant or approached significance. Most important of all, though, was the significant interaction between group and naming difficulty $\left[F(4,180)=4.45, M S_{\mathrm{e}}=2.62\right.$; Huynh-Feldt $\varepsilon=$ 1.0]. That is, the key effect observed above (interaction of naming difficulty, naming, and exposure) appears to result primarily from differences in the LON condition.

Third, we performed a series of contrasts to examine whether the LON group differed from the combined group (METO, ETON, and NEP) for each of our related dependent variables (evocation difficulty, vividness, and time). In addition, we conducted these analyses by difficulty level, in order to identify whether the effect in the LON group was primarily occurring for the easier-to-name odors. For evocation difficulty, there was a significant contrast of group $\times$ naming difficulty $\left[F(4,180)=5.64, M S_{\mathrm{e}}=\right.$ 1.22; Huynh-Feldt $\varepsilon=1.0]$. Both linear $[F(1,45)=9.39$, $\left.M S_{\mathrm{e}}=1.61\right]$ and quadratic $\left[F(1,45)=5.24, M S_{\mathrm{e}}=1.15\right]$ trends were significant for this interaction, indicating that the improvement in evocation in the LON group occurred primarily for the easier-to-name odors. For vividness, there was also a significant contrast of group $\times$ naming difficulty $\left[F(4,180)=3.67, M S_{\mathrm{e}}=1.36\right.$; Huynh-Feldt $\varepsilon=0.94]$. In this case, only the linear trend was significant for this interaction $\left[F(1,45)=8.13, M S_{\mathrm{e}}=1.49\right]$, indicating again that the improvement in vividness in the LON group was primarily for the easier-to-name odors. For time, the contrast of group $\times$ naming difficulty was not significant $(F=1.78)$. However, the linear trend for this interaction approached significance $[F(1,45)=3.75$, $\left.M S_{\mathrm{e}}=5.06, p=.059\right]$, indicating broadly the same effect as with the other two variables.

\section{Hedonic Deviation of the Image}

Hedonic deviation scores (see Experiment 1 for more details) were not significantly associated with evocation difficulty, vividness, or time, and so were analyzed separately, as they were in Experiment 1 (median $r=$ -.03 , range .45 to -.39 ; correlation between hedonic variable with the other three dependent variables at each level of difficulty [15 in total]). Using a three-way repeated measures ANOVA, with naming difficulty as the within-participants factor and exposure and naming as the between-participants factors, revealed a main effect of naming difficulty $\left[F(4,172)=7.20, M S_{\mathrm{e}}=0.78\right.$; Huynh-Feldt $\varepsilon=1.0]$. Overall, participants tended to give more divergent hedonic ratings as naming difficulty increased (see Table 4), as reflected in the significant linear $\left[F(1,43)=20.42, M S_{\mathrm{e}}=0.77\right]$ and quadratic $[F(1,43)=$ $\left.5.89, M S_{\mathrm{e}}=0.84\right]$ trends for this variable. However, there was also a main effect of naming $\left[F(1,43)=6.70, M S_{\mathrm{e}}=\right.$ $1.89]$, with less deviation from real odor hedonic ratings in participants who were provided with the odor names $(M=1.5)$ than in participants who were not $(M=2.0)$. There was also an interaction between these two factors $\left[F(4,172)=2.58, M S_{\mathrm{e}}=0.78\right.$; Huynh-Feldt $\left.\varepsilon=1.0\right]$, with apparently less deviation from the real odor hedonic ratings in the difficult-to-name odors, for those given names during training (see Table 4). This interpretation was partially supported by the marginally significant linear trend for this interaction $\left[F(1,43)=3.75, M S_{\mathrm{e}}=0.77\right.$, $p=.054]$.

\section{Participant Motivation, Olfactory Imagery, and Social Desirability}

There were no significant differences in self-reported olfactory imagery, social desirability, or motivation between conditions (ANOVA).

\section{Discussion}

Experiment 2 replicated the key finding from Experiment 1 , in that more-difficult-to-name odors are also reportedly more difficult to imagine. More importantly, the findings here suggest that learning odor names (1) improves the ease with which participants report evoking images, (2) improves the images' vividness, and (3) may shorten the time that participants spend attempting to evoke them. This performance enhancement was most ap- 

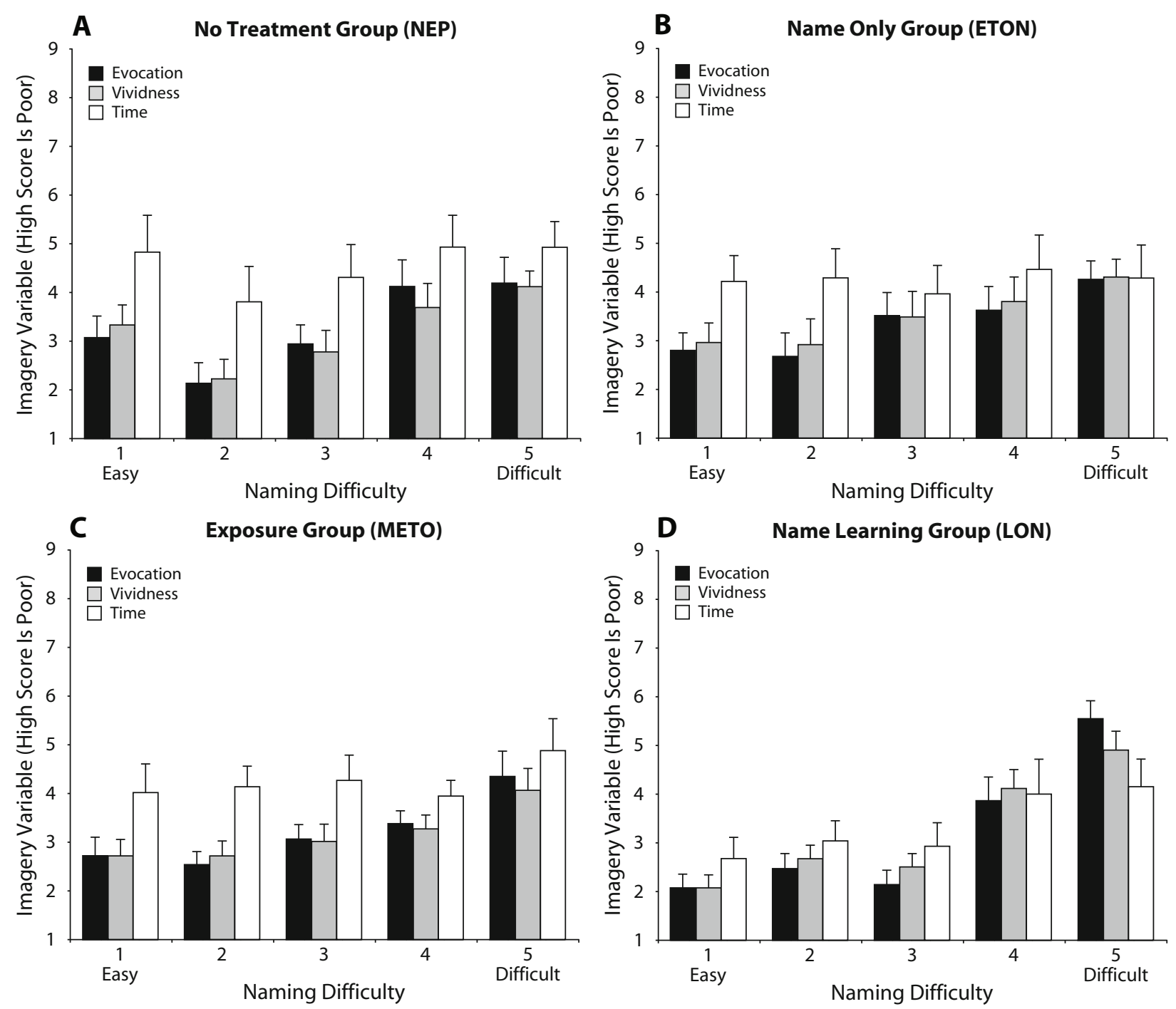

Figure 2: Mean imagery performance score (evocation, vividness, and time to form image; and $S E$ ) for the (A) no exposure (NEP), (B) exposure to odor names (ETON), (C) mere exposure to odors (METO), and (D) learning odor names (LON) conditions, by odornaming difficulty.

parent for the easy-to-name and moderately hard-to-name odors, but learning odor names did not have any obvious effect on those found difficult to name. The hedonic findings were less conclusive. Although training involving odor names appeared to benefit the accuracy with which participants assigned hedonic values to these stimuli, the benefit was present to a similar degree in the group exposed to odor names alone.

\section{GENERAL DISCUSSION}

Our primary aim was to explore the relationship between odor naming and self-report odor imagery. Experiment 1 revealed that increasing difficulty in odor naming was associated with increasing difficulty in image evocation, a relationship that did not obtain for common pictorial representations of the odors' source. In addition, a regression analysis by odor found that odor naming was the best predictor of difficulty of odor-image evocation, over and above the ability to name the odors' visual referents. Experiment 2 demonstrated that learning odor names improved self-reported imagery ability, but that mere exposure to the odors, their names, or no preexposure did not. This effect was most evident for the easier-to-name stimuli. Finally, Experiment 2 indicated that the hedonic tone of the olfactory images was more divergent from participants' real hedonic ratings for harder-to-name odors than for easier-to-name odors, and that participants with name training (names alone or odors and names) offered hedonic ratings more in agreement with those they later gave to the real stimuli. These findings represent the first demonstration of a substantial relationship between naming and self-report imagery ability, and in the discussion below, we examine the validity of this conclusion and its implications for understanding the mechanisms of olfactory imagery. 
Table 4

Absolute Hedonic Deviation (Real Odor Minus Image) by Naming

Difficulty Level for Each Group (Learning Odor Names [LON], Mere
Exposure to Odors [METO], Exposure to Odor Names [ETON], and No Exposure [NEP]) on Test in Experiment 2

\begin{tabular}{lcccc}
\hline & \multicolumn{5}{c}{ Mean Hedonic Deviation } \\
\cline { 2 - 5 } Odor-Naming Difficulty (Stimuli) & LON & METO & ETON & NEP \\
\hline $\begin{array}{l}\text { Level 1 [Easiest] } \\
\quad \text { (Vicks, baby powder, lemon) }\end{array}$ & 1.5 & 1.3 & 1.8 & 1.9 \\
$\begin{array}{l}\text { Level 2 } \\
\quad \text { (Vanilla, tomato sauce, peanut butter) }\end{array}$ & 1.2 & 1.7 & 1.3 & 1.4 \\
$\begin{array}{l}\text { Level 3 } \\
\quad \text { (Vegemite, coffee, shoe polish) }\end{array}$ & 1.1 & 2.3 & 1.4 & 2.3 \\
$\begin{array}{l}\text { Level 4 } \\
\quad(\text { Garlic, almond, oregano) }\end{array}$ & 1.4 & 1.9 & 1.8 & 2.0 \\
$\begin{array}{l}\text { Level 5 [Hardest] } \\
\quad(\text { Treacle, ginger, burnt wood) }\end{array}$ & 1.8 & 2.9 & 2.2 & 2.5 \\
$\begin{array}{l}\text { Overall mean } \\
\quad\end{array}$ & 1.4 & 2.0 & 1.7 & 2.0 \\
\hline
\end{tabular}

Asking participants to form a visual image of a persimmon might lead to the unwarranted conclusion that participants were rather poor at forming visual images until one raised the obvious objection that many participants do not know what a persimmon is. Similarly, here, one might be concerned that our results stemmed simply from choosing some hard-to-name objects. However, Experiment 1 demonstrated that the problem was not with the objects per se but with the modality in which they were experienced. Few participants had trouble identifying pictures of garlic, almonds, or shoe polish, but many participants, if not most, had trouble identifying them when the cue was an odor. Consequently, it is difficult to argue that these were hard-to-name objects in general; they were just hard-to-name objects in the olfactory modality. Similarly, when presented with the name during the imagery task (i.e., "imagine the smell of garlic" vs. "visualize a clove of garlic"), participants" olfactory imagery performance was primarily related to the difficulty in naming the odor, not its visual referent.

In the introduction, we briefly outlined why we thought that odor naming and odor imagery might be related. However, there is a further level to this argument that we did not allude to there. During the process of naming an odor, a participant forms a percept, and this then has to be linked to a name. During imagery, the name is presented, and this has to activate a memory of a percept. According to several contemporary accounts of odor perception (e.g., Haberly, 2001; Haberly \& Bower, 1989; Li \& Hertz, 2000; Wilson \& Stevenson, 2003), perception relies on a recognition process in which the pattern of stimulation from the olfactory receptors is matched to previously stored patterns of activation - odor memory. Thus, the odor percept resulting from a real olfactory stimulus probably involves a significant mnemonic component (Stevenson \& Boakes, 2003). Generating a name, then, involves activation of associations to semantic memory and the production of a name. Consequently, the processes used in going from a memory to a name (identification) and from a name to a memory (imagery) may be more closely aligned than one might imagine. Before offering a critical appraisal of this account, we start by examining the degree to which the data from these two experiments are consistent with it. We then turn to the parts that do not fit so well and the ambiguities that surround the associative account, especially its neuroanatomical underpinning.

Key support for our theoretical stance is provided by the finding in Experiments 1 and 2 that hard-to-name odors are more difficult to evoke than easier-to-name odors. In essence, the stronger the associative link from an odor memory to its name (as evidenced by naming the odor), the stronger the reciprocal link is too (as evidenced by reports of its imagineability). Obviously, hard-to-name odors are also likely to be less familiar and consequently more disliked (e.g., Lawless \& Cain, 1975), but the regression analysis in Experiment 1 offers some support for our contention that the primary difference results from difficulty in naming, not the odor's general unfamiliarity nor, for that matter, the difficulty that participants might have in identifying its visual referent. Further support is offered by the finding in Experiment 2 that it was only odor-name learning that improved imagery performance. That is, strengthening the association between an odor memory and its name (i.e., naming the odor) has the effect of strengthening the reciprocal connection too (i.e., its imagineability). We must, however, qualify this picture with a number of interesting observations that may suggest some revision of this account.

First, we had expected that learning odor names would have generally improved imagery, yet this was not the case. Most of the benefit occurred for the easier-to-name stimuli. Second, in Experiment 1, participants in the olfactory condition, who received the odor-naming phase first, appeared to have benefited from this preexposure, yet participants were not given feedback as to the correctness of their responses, nor did they differ in the number of odors that they correctly identified, in comparison with participants who received the naming test second. Third, the hedonic variable, a further (albeit new) measure of imagery quality, did not behave as we had expected. In Experiment 1, there was no clear relationship with odor-naming difficulty, although a linear relationship was observed in Experiment 2. More important, though, was the finding that having prior exposure to names (with or without odors) diminished the 
difference between the real and imagined hedonic ratings. We deal with each of these points in turn.

The finding that the easier-to-name odors benefited most (in terms of their imagineability) from odor-name learning might reflect the degree of training needed to strengthen the memory-name association to a level at which it starts to benefit its reciprocal association. If the amount of training required was substantial, then any effect would manifest first for the easier-to-name stimuli, as we observed. This would be fairly simple to test, by manipulating the level of odor-name training. However, can this interpretation be correct, given the finding from Experiment 1 that imagery performance was reportedly better in participants who had attempted to name the odors first? Even if attempting to generate a name for each odor is equivalent to limited name training, an effect within one trial contradicts the notion that substantial training may be needed to benefit the imagineability of easier-to-name odors. One solution is to suggest that the degree of name learning needed to improve imagery performance is relatively slight for easy-to-name odors, but that progressive improvements for more difficult-to-name odors are not linear. Yet again, this would be fairly easy to test experimentally.

The hedonic data raise a different problem. If we had found that the difference between actual and imagined hedonic ratings was smallest in just the LON condition, our interpretation could have been straightforward, because it would have been consistent with our other findings. However, the presence of an effect in both groups exposed to names (LON and ETON) implies one of two possibilities. The first possibility, the parsimonious one, is that a common process underpinned an effect in both groups, suggesting that prior experience with the names (and thus not names and odor) causes this effect. The second possibility is that there was a different cause in each group. In respect to the second point, it is important to recollect that the ETON group was asked to judge whether they liked or disliked the words during their training phase. As noted in the Method section, we were careful to stipulate that this task pertained to the words themselves, but we could not avoid testing participants in the same room (with much odor-related paraphernalia) and having them sign the same consent form as participants in the other groups - that is, they knew that, at some level, the task related to smell. On this basis, the ETON participants might have reflected upon how much they liked/disliked the odor to which the word referred. This may have enabled them to give more accurate judgments in the imagery phase of the study. Finally, on a more general point, utilizing hedonic differences as a measure of imagery ability is a new technique, so there is little data, as yet, with which to judge its reliability or validity. Nevertheless, we included it, because the affective component of olfactory imagery has been little explored, yet the emotive power of odors is well documented (e.g., Rouby \& Bensafi, 2002).

Although the findings here are supportive of an associative account of odor imagery, the account itself has a problem in explaining why the link between names and odors should be so poor. A classic response in this regard is to claim that anatomically, olfactory and semantic memory are relatively remote and are sparsely interconnected (see Stevenson \& Case, 2005b). However, we know of no data that strongly support this suggestion, and it has been criticized on just such grounds (e.g., Lorig, 1999). Even if the anatomically based account were true, it does not address our key assumption that if communication is poor in one direction, then it will be poor in the other. Until we know more about the neural networks that subserve associative pathways used in odor naming and imagery, these assumptions will remain just that - assumptions. Nevertheless, the data here do suggest that naming and imagery are connected in ways not previously identified.

\section{AUTHOR NOTE}

We thank Margery Aylett and Simon Mingo for assistance in conducting these experiments. This work was supported by the Australian Research Council. Correspondence concerning this article should be addressed to R. J. Stevenson, Department of Psychology, Macquarie University, Sydney, NSW2109, Australia (e-mail: richard.stevenson@, psy.mq.edu.au).

\section{REFERENCES}

Algom, D., \& Cain, W. S. (1991). Remembered odors and mental mixtures: Tapping reservoirs of olfactory knowledge. Journal of Experimental Psychology: Human Perception \& Performance, 17, 1104-1119.

Algom, D., Marks, L. E., \& CaIN, W. S. (1993). Memory psychophysics for chemosensation: Perceptual and mental mixtures of odor and taste. Chemical Senses, 18, 151-160.

Ashton, R., \& White, K. D. (1980). Sex differences in imagery vividness: An artefact of the test. British Journal of Psychology, 71, 35-38.

Bensafi, M., Porter, J., Pouliot, S., Mainland, J., Johnson, B., Zelano, C., ET AL. (2003). Olfactomotor activity during imagery mimics that during perception. Nature Neuroscience, 6, 1142-1144.

Bensafi, M., Pouliot, S., \& Sobel, N. (2005). Odorant-specific patterns of sniffing during imagery distinguish "bad" and "good" olfactory imagers. Chemical Senses, 30, 521-529.

BROWER, D. (1947). The experimental study of imagery: II. The relative predominance of various imagery modalities. Journal of General Psychology, 37, 199-200.

CAIN, W. S. (1979). To know with the nose: Keys to odor identification. Science, 203, 468-470.

Cain, W. S., De Wijk, R., Lulejian, C., Schiet, F., \& See, L.-C. (1998). Odor identification: Perceptual and semantic dimensions. Chemical Senses, 23, 309-326.

Cain, W. S., \& PotTs, B. C. (1996). Switch and bait: Probing the discriminative basis of odor identification via recognition memory. Chemical Senses, 21, 35-44.

Crosley, C. J., \& Dhamoon, S. (1983). Migrainous olfactory aura in a family. Archives of Neurology, 40, 459.

Crowne, D. P., \& Marlow, D. (1960). A new scale of social desirability independent of psychopathology. Journal of Consulting Psychology, 24, 349-354.

Daly, D. (1958). Uncinate fits. Neurology, 8, 250-260.

Desor, J. A., \& Beauchamp, G. K. (1974). The human capacity to transmit olfactory information. Perception \& Psychophysics, 16, 551-556.

DJordjevic, J., Zatorre, R., \& Jones-Gotman, M. (2004). Effects of perceived and imagined odors on taste detection. Chemical Senses, 29, 199-208.

Djordjevic, J., Zatorre, R., Petrides, M., \& Jones-Gotman, M. (2004). The mind's nose: Effects of odor and visual imagery on odor detection. Psychological Science, 15, 143-148.

Gilbert, A. N., Crouch, M., \& Kemp, S. E. (1998). Olfactory and visual mental imagery. Journal of Mental Imagery, 22, 137-146. 
Haberly, L. B. (2001). Parallel-distributed processing in olfactory cortex: New insights from morphological and physiological analysis of neuronal circuitry. Chemical Senses, 26, 551-577.

Haberly, L. B., \& Bower, J. M. (1989). Olfactory cortex: Model circuit for study of associative memory? Trends in Neurosciences, 12, 258-264.

Hurovitz, C. S., Dunn, S., Domhoff, G. W., \& Fiss, H. (1999). The dreams of blind men and women: A replication and extension of previous findings. Dreaming, 9, 183-193.

Jonsson, F. U., Tchekhova, A., Lonner, P., \& Olsson, M. J. (2005). A metamemory perspective on odor naming and identification. Chemical Senses, 30, 353-365.

Kosslyn, S. M., Ganis, G., \& Thompson, W. L. (2003). Mental imagery: Against the nihilistic hypothesis. Trends in Cognitive Sciences, 7, 109-111.

LAWLESS, H. T. (1997). Olfactory psychophysics. In G. K. Beauchamp \& L. Bartoshuk (Eds.), Tasting and smelling (pp. 125-175). San Diego: Academic Press.

LAWLESS, H. T., \& CAIN, W. S. (1975). Recognition memory for odors Chemical Senses \& Flavour, 1, 331-337.

LAWLESS, H. T., \& ENGEN, T. (1977). Associations to odors: Interference, mnemonics and verbal labelling. Journal of Experimental Psychology: Human Learning \& Memory, 3, 52-59.

Li, Z., \& Hertz, J. (2000). Odour recognition and segmentation by a model olfactory bulb and cortex. Network: Computational Neural Systems, 11, 83-102.

Lindauer, M. S. (1969). Imagery and sensory modality. Perceptual \& Motor Skills, 29, 203-215.

LORIG, T. S. (1999). On the similarity of odor and language perception. Neuroscience \& Biobehavioral Reviews, 23, 391-398.

Lyman, B. J., \& McDaniels, M. A. (1990). Memory for odors and odor names: Modalities of elaboration and imagery. Journal of Experimental Psychology: Learning, Memory, \& Cognition, 16, 656-664.

MARKs, D. F. (1973). Visual imagery differences in the recall of pictures. British Journal of Psychology, 64, 17-24.

Marsella, A. J., \& QuiJAno, W. Y. (1974). A comparison of vividness of mental imagery across different sensory modalities in Filipinos and Caucasian-Americans. Journal of Cross-Cultural Psychology, 5, 451-465.

McKelvie, S. J. (1995). The VVIQ as a psychometric test of individual differences in visual imagery vividness: A critical quantitative review and plea for direction. Journal of Mental Imagery, 19, 1-106.

Richardson, J. T. E. (1999). Imagery. Hove, U.K.: Psychology Press.

Rouby, C., \& Bensafi, M. (2002). Is there a hedonic dimension to odors? In C. Rouby, B. Schaal, D. Dubois, R. Gervais, \& A. Holley (Eds.), Olfaction, taste and cognition (pp. 140-159). Cambridge, U.K.: Cambridge University Press.

SEgal, S. J. (1971). Processing of the stimulus in imagery and perception. In S. J. Segal (Ed.), Imagery: Current cognitive approaches (pp. 69-100). New York: Academic Press.

Sheehan, P. W. (1967). A shortened form of Betts' Questionnaire upon mental imagery. Journal of Clinical Psychology, 23, 386-389.

SteVenson, R. J., \& Boakes, R. A. (2003). A mnemonic theory of odor perception. Psychological Review, 110, 340-364.

Stevenson, R. J., \& Case, T. I. (2005a). Olfactory dreams: Phenomenology, relationship to volitional imagery and odor identification. Imagination, Cognition \& Personality, 24, 69-90.

Stevenson, R. J., \& CASE, T. I. (2005b). Olfactory imagery: A review. Psychonomic Bulletin \& Review, 12, 244-264.

Stevenson, R. J., \& Prescott, J. (1997). Judgments of chemosensory mixtures in memory. Acta Psychologica, 95, 195-214.

Stevenson, R. J., \& Repacholi, B. M. (2003). Age-related changes in children's hedonic response to male body odor. Developmental Psychology, 39, 670-679.

White, K. D., Ashton, R., \& Law, H. (1978). The measurement of imagery vividness: Effects of format and order on Betts' Questionnaire upon mental imagery. Canadian Journal of the Behavioral Sciences, 10, 68-78.

Wilson, D. A., \& Stevenson, R. J. (2003). The fundamental role of memory in odor perception. Trends in Neurosciences, 26, 243-248.

(Manuscript received October 16, 2005; revision accepted for publication February 9, 2006.) 